\title{
Cosmic radiation exposure of biological test systems during the EXPOSE-R mission
}

\author{
Thomas Berger ', Michael Hajek ${ }^{2,3}$, Pawel Bilski $^{4}$ and Günther Reitz' \\ ${ }^{1}$ Radiation Biology Department, German Aerospace Center (DLR), Institute of Aerospace Medicine, Cologne, Germany \\ e-mail: thomas.berger@dlr.de \\ ${ }^{2}$ Vienna University of Technology, Institute of Atomic and Subatomic Physics ( ATI), Vienna, Austria \\ ${ }^{3}$ International Atomic Energy Agency, Division of Radiation, Transport and Waste Safety, 1400 Vienna, Austria \\ ${ }^{4}$ Department of Radiation Physics and Dosimetry, Institute of Nuclear Physics (IFJ), Krakow, Poland
}

\begin{abstract}
In the frame of the EXPOSE-R mission outside the Russian Zvezda Module of the International Space Station (ISS) passive thermoluminescence dosimeters were applied to measure the radiation exposure of biological samples. The detectors were located beneath the sample carriers to determine the dose levels for maximum shielding. The dose measured beneath the sample carriers varied between $317 \pm 10$ and $230 \pm 2$ $\mathrm{mGy}$, which amount to an average dose rate of $381 \pm 12$ and $276 \pm 2 \mu \mathrm{Gy} \mathrm{d}^{-1}$. These values are close to those assessed for the interior of the ISS and reflect the high shielding of the biological experiments within the EXPOSE-R facility. As a consequence of the high shielding (several $\mathrm{g} \mathrm{cm}^{-2}$ ), the biological samples were predominantly exposed to galactic cosmic heavy ions and trapped protons in the Earth's radiation belts, whereas the trapped electrons did not reach the samples.

Received 31 July 2014, accepted 29 September 2014, first published online 30 October 2014
\end{abstract}

Key words: dosimetry, EXPOSE-R, passive radiation detectors, space radiation, thermoluminescence detectors.

\section{Introduction}

The radiation environment in space is the most complex natural radiation field. It mainly consists of highly energetic charged particles from protons to iron ions (galactic cosmic rays), particles trapped by the Earth's magnetic field (protons, electrons and a few heavier ions) and particles emitted in so-called solar particle events during periods of high solar activity (mainly protons) (Hovestadt et al. 1978; Facius \& Reitz 2006; Reitz 2008). Inside a spacecraft the radiation field becomes even more complex than externally, because the primary particles interact with the atoms of the structural materials and produce secondary radiation (Benton \& Benton 2001). Therefore, for any biological experiment in space the knowledge of the actual doses received by the test systems is required (Goossens et al. 2006; Vanhavere et al. 2008).

The EXPOSE-R facility, which was attached to the external side of the Russian Zvezda Module of the International Space Station (ISS) for 682 days (Rabbow et al. 2014, this issue), housed a variety of organisms (from microorganisms to plant seeds) and organic chemical compounds. The samples were exposed to selected space conditions (vacuum, Solar UV, cosmic rays and varying temperatures) and their reactions were analysed after retrieval. EXPOSE-R was launched by Progress 31P on November 26, 2008 and returned to Earth on March 09, 2011. The absorbed dose received by the biological samples contained in the EXPOSE-R experiment carrier was measured as part of the Response of Organisms to Space Environment (ROSE) experiment applying passive thermoluminescence detectors (TLDs). Passive TLDs have been applied for radiation measurements on board various space stations and Space Shuttle missions since the beginning of the space age (Reitz et al. 2005, 2009; Berger 2008; Hajek et al. 2008; Berger et al. 2013). Their small size of a few $\mathrm{mm}^{3}$ and low mass (around $20 \mathrm{mg}$ ) allow affixing them close to the samples of interest. During the EXPOSE-R mission, TLDs were accommodated at different sites in close vicinity to the biological specimens. The data acquired within the experiment provided reference doses for the ROSE experiments (Rabbow et al. 2014, this issue). In addition to the passive radiation detectors placed beneath the sample carriers, the time profile beneath very low shielding was measured with the Radiation Risks Radiometer-Dosimeter (R3DR) (Dachev et al. 2014, this issue).

\section{Materials and methods}

\section{Passive radiation detectors}

TLDs made of ${ }^{7} \mathrm{LiF}$ with $\mathrm{Mg}$ and $\mathrm{Ti}$ dopants, provided by Thermo Fisher Scientific Inc. (former Harshaw Chemical Co.), Solon, OH, USA, under the trade name TLD-700, and TLD Poland, Krakow, Poland, under the trade name MTS-7, were used as passive radiation dosimeters. The energy deposited by ionizing radiation in the TLDs is stored at defect centres in the crystal lattice. During readout in the laboratory, the stored energy is released as light upon heating. When the light intensity is plotted over temperature yielding the so-called glow curve, the amplitude or region of interest (ROI) of specific peaks is proportional to the absorbed dose, thus enabling TLD utilization in radiation dosimetry upon calibration in gamma-ray fields (Berger \& Hajek 2008; Bilski et al. 2011). 
As an example, Fig. 1 shows a glowcurve from one TLD measured after the EXPOSE-R experiment (triangles) and, for comparison purposes, the glowcurve from the same TLD after gamma calibration with $100 \mathrm{mGy}$ of ${ }^{137} \mathrm{Cs}$ (circles).

While the history of space radiation dosimetry showed a high degree of disagreement among the results acquired by different laboratories, the convergence of measurements reported in the present manuscript is the result of a long-term harmonization process, which could be achieved through intercomparison experiments such as the ICCHIBAN campaign initiated in the early 2000s (Yasuda et al. 2006). The high level of consistency in the doses evaluated independently by three different groups, each applying its own experimental protocols, clearly demonstrates the reliability of the generated data. The protocols in use for TLD annealing, readout and calibration are summarized in Table 1. Further investigations were related to the long-term stability of the TL signal with due consideration of the different temperature regimes encountered during on-board storage (Bilski et al. 2013). TL efficiency for heavy ions encountered in the space radiation environment with respect to gamma rays was assessed in Berger \& Hajek (2008) and Bilski et al. (2011). Further on data comparison during actual space experiments Bilski et al. (2011) and Berger et al. $(2012,2013)$ proved the reliability and consistency of the data gathered by the three research groups. As indicated by these long-term Earth and Space-based studies, the acquired data are fully compatible. Throughout the present paper, all doses are given in terms of absorbed dose in water.

\section{Space flight experiment}

\section{EXPOSE-R overview}

The EXPOSE-R facility was launched by Progress 31P on November 26, 2008 and returned to Earth on March 09, 2011. It was mounted outside the Zvezda module of the ISS on March 10, 2009 (see Fig. 2) and recovered from outside on January 21, 2011. This leads to total mission duration of 833 days, with an exposure outside the ISS of 682 days.

To obtain the actual doses received by the biological samples of EXPOSE-R to the best extent possible, the passive radiation detectors were placed close to the samples. TLDs of the types TLD-700 (ATI and DLR) and MTS-7 (IFJ) were enclosed in acrylic glass holders with a dimension of $28 \times 28 \times 8.5 \mathrm{~mm}$ and placed beneath the biological sample carriers in Tray- 2 and Tray-3 of the EXPOSE-R facility (Fig. 3). A total number of eight holders were positioned beneath the dark samples in Tray-2, while for Tray-3 six detector holders were positioned beneath the samples (Fig. 4). The lower left part of Tray-3 (shown in Fig. 4) houses the R3DR instrument (Dachev et al. 2014, this issue).

After return of EXPOSE-R to ground, the TLDs were extracted from the facility at DLR, Cologne, Germany, and distributed to the project partners (ATI, Vienna, Austria; IFJ, Krakow, Poland) for processing and evaluation. TLD readout and data acquisition followed the protocols outlined in Table 1. Due to the fact that TLDs are integrating devices,

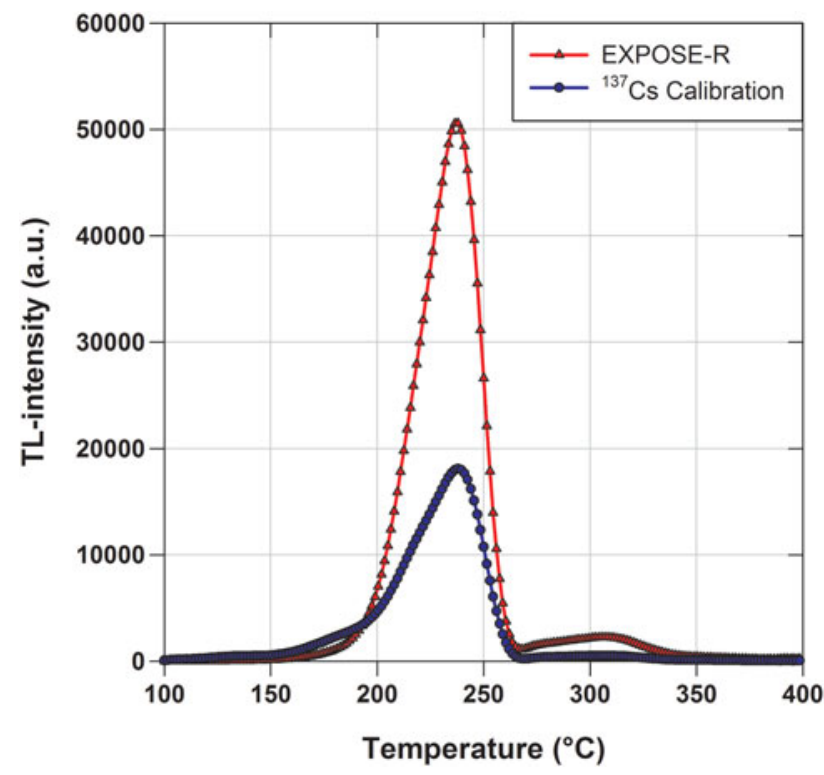

Fig. 1. Example of glowcurves measured by DLR after the EXPOSE-R mission (triangles) and the respective glowcurve after calibration with $100 \mathrm{mGy}{ }^{137} \mathrm{Cs}$ (circles).

only the total absorbed dose accumulated over the entire duration of the EXPOSE-R mission was measured.

\section{EXPOSE-R temperature}

In combination with potential temperature variations, the long duration of the EXPOSE-R experiment may be considered to give rise to fading effects in TLDs. The temperature inside the EXPOSE-R facility was monitored with numerous sensors. The temperature was fluctuating, with the maximum and minimum temperatures being +49.47 and $-24.65^{\circ} \mathrm{C}$, respectively. The calculated mission-average temperature was $+19{ }^{\circ} \mathrm{C}$ (Rabbow et al. 2014). The performance of TLDs used in EXPOSE-R was recently studied in a long-term fading experiment (Bilski et al. 2013). During these investigations, TLDs were stored at different temperatures for more than 1 year, following exposure to gamma rays and neutrons. The storage of TLDs in conditions of naturally fluctuating temperature (minimum $+14{ }^{\circ} \mathrm{C}$, maximum $+28^{\circ} \mathrm{C}$ ) with an average of $+18.4{ }^{\circ} \mathrm{C}$ revealed high stability of TLDs, with fading being negligible. Although the maximum temperature of the EXPOSE-R experiment was somewhat higher, the average values were almost identical, thus that significant fading may be excluded.

\section{EXPOSE-R local shielding}

For the interpretation of the measured dose rate within the EXPOSE-R mission, it is essential to have a baseline estimation of the 'local shielding' seen by the samples located (as given in Fig. 3) below the biological species. The biological samples in the EXPOSE-R facility were protected at the top layer (see Fig. 4) with various optical filter systems (made either of Suprasil or $\mathrm{MgF}_{2}$ ) with thicknesses of at least $1 \mathrm{~cm}$ leading to a shielding at the top of the samples of around 
Table 1. Readout procedures for the thermoluminescence detectors applied by the collaborators: DLR, Cologne, Germany; ATI, Vienna, Austria; IFJ, Krakow, Poland

\begin{tabular}{|c|c|c|c|}
\hline & $\mathrm{DLR}^{\mathrm{a}}$ & $\mathrm{ATI}^{\mathrm{a}}$ & $\mathrm{IFJ}^{\mathrm{b}}$ \\
\hline TLD manufacturer & Thermo, USA & Thermo, USA & TLD Poland \\
\hline Annealing temperature/time & $\begin{array}{l}400^{\circ} \mathrm{C} / 1 \mathrm{~h} \\
+100^{\circ} \mathrm{C} / 2 \mathrm{~h}\end{array}$ & $400^{\circ} \mathrm{C} / 1 \mathrm{~h}$ & $\begin{array}{l}400^{\circ} \mathrm{C} / 1 \mathrm{~h} \\
+100^{\circ} \mathrm{C} / 2 \mathrm{~h}\end{array}$ \\
\hline Cooling & Slow & Very slow (24 h) & Fast \\
\hline Pre-readout treatment & - & $120^{\circ} \mathrm{C} / 30 \mathrm{~min}$ & $120^{\circ} \mathrm{C} / 30 \mathrm{~min}$ \\
\hline Reader type & Harshaw 5500 with Hamamatsu PMT & TLD-DAT.II with EMI PMT & RA-94 with EMI PMT \\
\hline Heating during readout & Gas, $5^{\circ} \mathrm{C} \mathrm{s}^{-1}$, end temp. $400{ }^{\circ} \mathrm{C}$ & Contact, $5^{\circ} \mathrm{C} \mathrm{s}^{-1}$ end temp. $480^{\circ} \mathrm{C}$ & Contact, $10^{\circ} \mathrm{C} \mathrm{s}^{-1}$ end temp. $400^{\circ} \mathrm{C}$ \\
\hline Neutral gas & Nitrogen & Nitrogen & Argon \\
\hline Calibration source & Cs-137 & Co-60 & Cs-137 \\
\hline Quantification of TL signal & Peak height & Peak height & ROI \\
\hline
\end{tabular}

${ }^{a} A T I$ and DLR applied TLD-700 detectors manufactured by Thermo (USA) with a dimension of $3.2 \times 3.2 \times 0.9 \mathrm{~mm}$.

${ }^{\mathrm{b}}$ IFJ applied MTS-7 detectors manufactured by TLD Poland with a diameter of $4.5 \mathrm{~mm}$ and a thickness of $0.9 \mathrm{~mm}$.

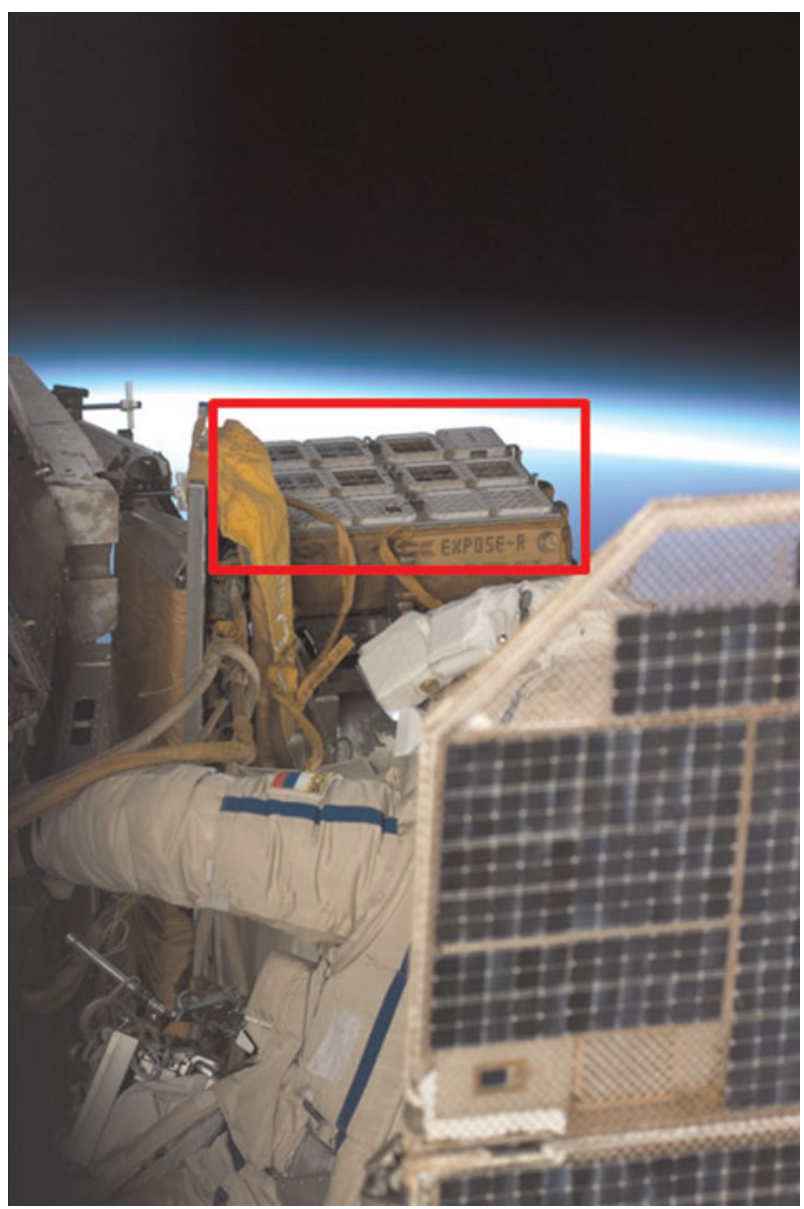

Fig. 2. EXPOSE-R experiment carrier (framed) attached to the outside of the Russian Zvzeda module.

$2.2-3.1 \mathrm{~g} \mathrm{~cm}^{-2}$. This shielding is already sufficient to shield the lower energetic trapped electrons, which exponentially decrease with increasing shielding depth (see also Fig. 5 in Berger et al. (2012)). The acrylic glass holders with the TLDs were located below the relevant sample carriers, made out of aluminium, which add an additional $\sim 1 \mathrm{~g} \mathrm{~cm}^{-2}$ to the shielding from above. Further, the EXPOSE-R facility was mounted

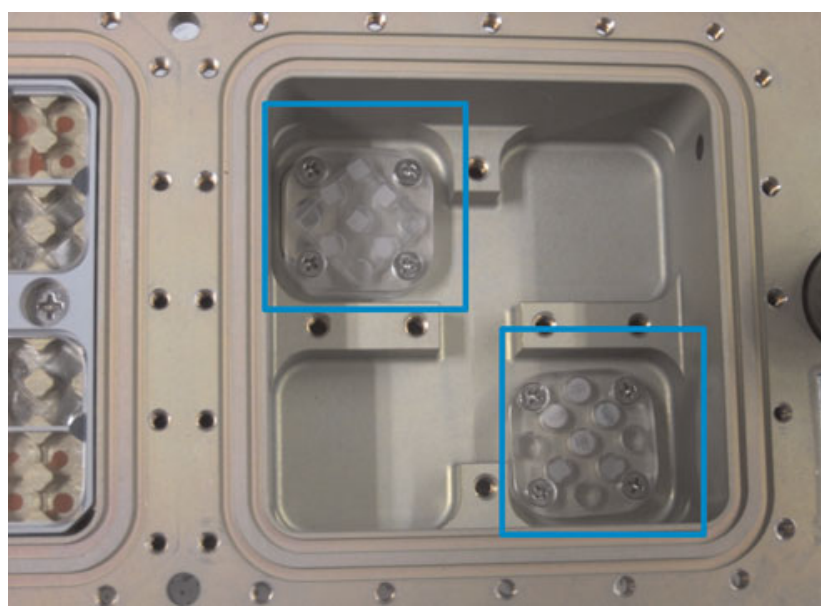

Fig. 3. TLDs in acrylic glass holders installed beneath the ROSE dark samples in Tray-2 of the EXPOSE-R facility.

outside the Russian Zvezda module (as seen in Fig. 1), adding additional shielding for both the radiation detectors and the biological samples for an almost $2 \pi\left(180^{\circ}\right)$ geometry from below. Additional shielding is provided 'locally' by the walls of the EXPOSE-R facility.

\section{Results}

In order to study the absorbed dose from ionizing radiation during the EXPOSE-R experiment, TLDs of the types ${ }^{7} \mathrm{LiF}$ : $\mathrm{Mg}$, Ti were contained in acrylic glass holders below the biological sample carriers (see Figs. 3 and 4). The missionintegrated absorbed dose beneath the biological sample carriers is given for each position within Tray- 2 and Tray- 3 of the EXPOSE-R facility in the columns 2-4 of Table 2 for the respective research groups. Further on the absorbed dose rate in $\mu \mathrm{Gy} \mathrm{d}^{-1}$ is given in columns $5-7$ of Table 2 .

Due to the fact, that TLDs are integrating detectors the daily dose rate is based on the overall mission duration of 833 days. One has to take into account, if looking at the dose rate data, that the facility was only exposed for 682 days outside the 


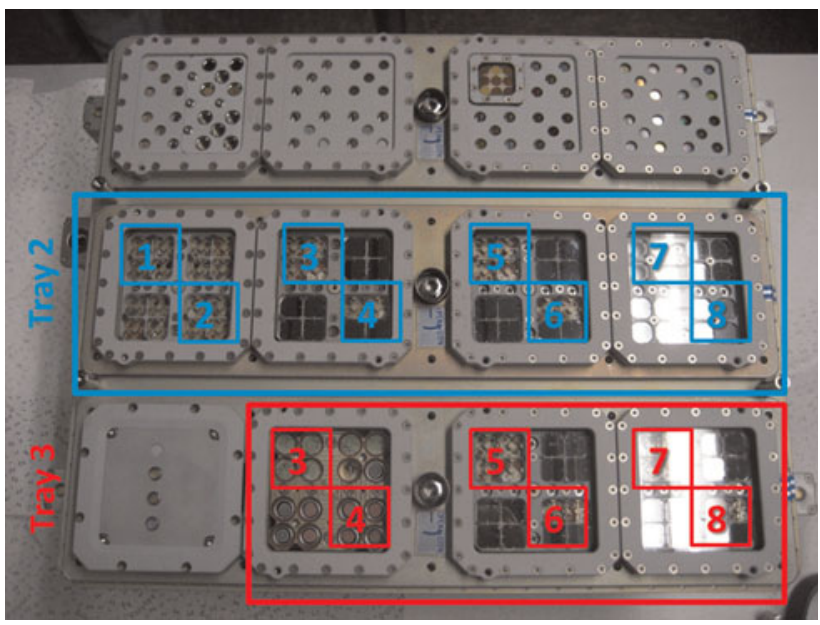

Fig. 4. Location of the measurement sites inside of EXPOSE-R in Tray-2 and Tray-3.

station with additional 151 days either inside the ISS (in the Russian Part of the ISS) or on the way to or from the ISS. Therefore the daily absorbed dose values include contributions from $82 \%$ outside and $18 \%$ inside exposure and are therefore to be seen as lower limits for the outside exposure. All the given uncertainties correspond to one standard deviation of the mean.

Unfortunately, no background control dosimeters, which would have stayed inside the ISS during the outside exposure, were available for the EXPOSE-R mission. One could, of course, take measured data from inside the Russian Modules as 'reference data' to be subtracted from the overall mission dose to calculate the dose rate for the outside exposure. This is, however, not an easy approach to follow. The absorbed dose values inside the Russian Segment of the ISS can vary tremendously depending on detector location, reflecting the different local shielding conditions. Absorbed dose measured with the active radiation detector DB-8 in the Russian Service Module was shown to vary between 200 and 360 $\mu \mathrm{G} \mathrm{d}^{-1}$ (see Fig. 1 in Lishnevskii et al. (2010) for the end of the year 2008 when the EXPOSE-R facility was stored in the Russian Segment of the ISS). Data measured with passive radiation detectors (TLDs) at various parts of the Russian Segment showed variations between 150 to almost $400 \mu \mathrm{Gy} \mathrm{d}^{-1}$ for the period of the EXPOSE-R mission (Welton et al. 2012). This high variation is supported by later measurement inside the Russian module performed by DLR resulting in dose variations between 240 and $340 \mu \mathrm{Gy} \mathrm{d}^{-1}$ (Pálfalvi et al. 2014). It is interesting to note, however, that the daily dose measured within the EXPOSE-R experiment is very close to the data given for measurements inside the Russian Segment of the ISS.

The relevant mission absorbed dose values, separated for the three research groups are further provided in Fig. 5(a) for the Tray-2 detectors and in Fig. 5(b) for the Tray-3 detectors.

For Tray-2 the total mission dose varies between $317 \pm 10$ $\mathrm{mGy}$ at Position 1 and $239 \pm 9 \mathrm{mGy}$ at Position 8 (Fig. 5(a)). The mission dose values show a decrease from Positions 1 to 4 followed by an almost constant mission dose for the
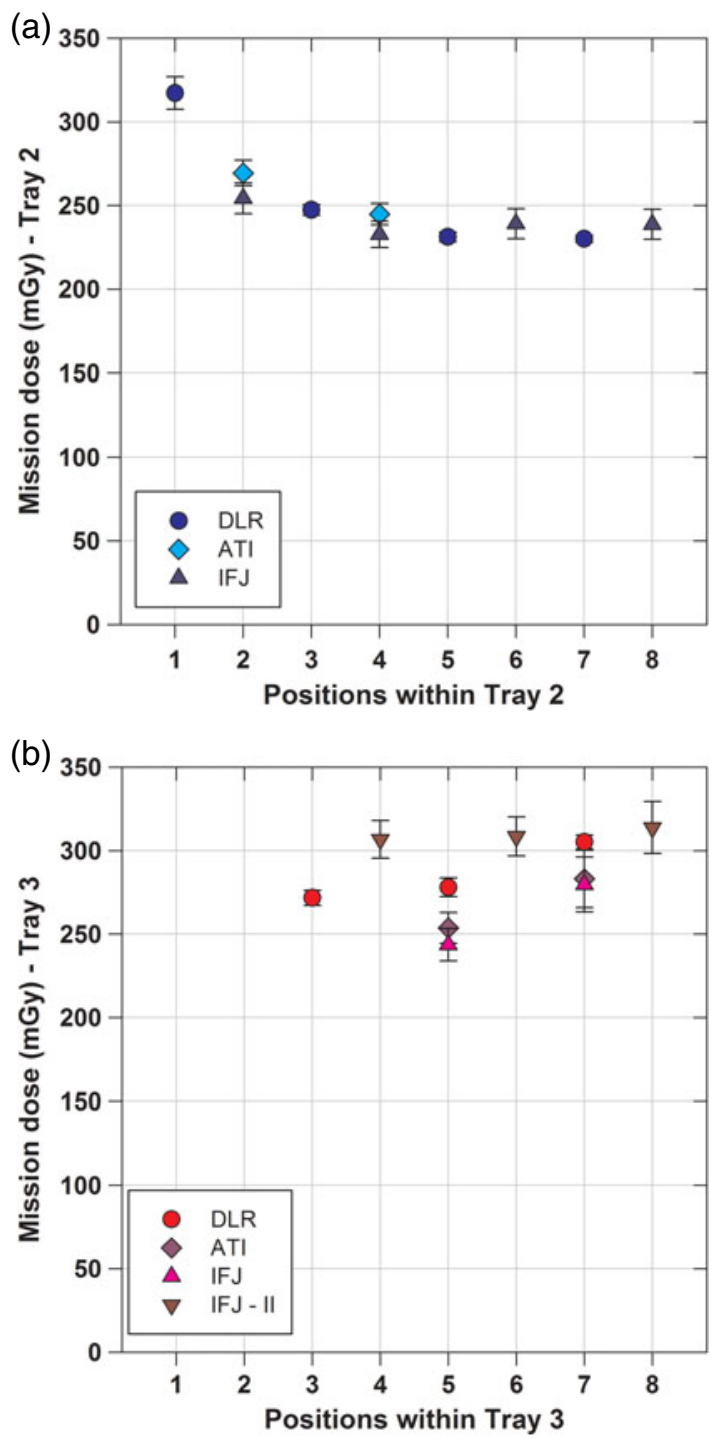

Fig. 5. Mission absorbed dose in mGy measured beneath the dark samples at (a) eight positions in Tray-2 and (b) six positions in Tray-3 of the EXPOSE-R facility.

Positions 4-8. The decrease can be explained by the local shielding of at one hand the EXPOSE-R facility itself and on the other hand by the respective shielding given from the Russian Zvezda module.

For Tray-3 (Fig. 5(b)) we see a slight increase in the mission absorbed dose for the odd number Positions 3, 5 and 7 with maximum dose values of $305 \pm 4 \mathrm{mGy}$. The even number Positions 4, 6 and 8 (See IFJ-II data in Fig. 5(b)) included detectors which were mounted very close to the outer wall of the EXPOSE-R facility, thereby seeing less shielding than the odd number Positions and resulting in mission dose values of up to $314 \pm 16 \mathrm{mGy}$.

\section{Discussion}

The data provided by the passive radiation detectors within the EXPOSE-R mission, positioned beneath the dark sample 
Table 2. Mission absorbed dose rates in mGy (columns 2-4) and daily absorbed dose rate (columns 5-7) measured with TLDs at eight positions within Tray-2 and at six positions within Tray-3 of the EXPOSE-R facility

\begin{tabular}{|c|c|c|c|c|c|c|}
\hline \multirow[b]{2}{*}{ TRAY-2 positions } & \multicolumn{3}{|c|}{ Absorbed mission dose (mGy) } & \multicolumn{3}{|c|}{ Absorbed dose rate $\left(\mu \mathrm{Gy} \mathrm{d}^{-1}\right)$} \\
\hline & DLR & ATI & IFJ & DLR & ATI & IFJ \\
\hline 1 & $317 \pm 10$ & & & $381 \pm 12$ & & \\
\hline 2 & & $270 \pm 8$ & $254 \pm 9$ & & $323 \pm 9$ & $305 \pm 11$ \\
\hline 3 & $247 \pm 3$ & & & $297 \pm 4$ & & \\
\hline 4 & & $245 \pm 6$ & $233 \pm 8$ & & $294 \pm 8$ & $279 \pm 9$ \\
\hline 5 & $231 \pm 3$ & & & $278 \pm 3$ & & \\
\hline 6 & & & $239 \pm 9$ & & & $287 \pm 11$ \\
\hline 7 & $230 \pm 2$ & & & $276 \pm 2$ & & \\
\hline 8 & & & $239 \pm 9$ & & & $287 \pm 11$ \\
\hline TRAY-3 positions & DLR & ATI & IFJ & DLR & ATI & IFJ \\
\hline 3 & $272 \pm 5$ & & & $326 \pm 5$ & & \\
\hline 4 & & & $307 \pm 11$ & & & $368 \pm 14$ \\
\hline 5 & $278 \pm 6$ & $254 \pm 9$ & $244 \pm 10$ & $334 \pm 7$ & $304 \pm 11$ & $292 \pm 12$ \\
\hline 6 & & & $308 \pm 12$ & & & $370 \pm 14$ \\
\hline 7 & $305 \pm 4$ & $283 \pm 17$ & $280 \pm 16$ & $366 \pm 5$ & $340 \pm 21$ & $336 \pm 20$ \\
\hline 8 & & & $314 \pm 16$ & & & $377 \pm 19$ \\
\hline
\end{tabular}

carriers showed mission absorbed dose values between 317 $\pm 10 \mathrm{mGy}(\max )$ and $230 \pm 2 \mathrm{mGy}(\mathrm{min})$ for Tray-2 and between $314 \pm 16 \mathrm{mGy}(\max )$ and $244 \pm 10 \mathrm{mGy}(\mathrm{min})$ for Tray- 3 for the whole mission duration of 833 days. The dose values vary over the Trays and over the positions within the Trays, reflecting thereby the local shielding environment of the EXPOSE-R facility and the respective Zvezda module of the ISS.

If one looks at the daily dose values based on the overall mission duration of 833 days the daily absorbed dose values measured beneath the dark samples of the EXPOSE-R facility would vary between $381 \pm 12 \mu \mathrm{Gy} \mathrm{d}^{-1}(\max )$ and $276 \pm 2$ $\mu \mathrm{Gy} \mathrm{d}^{-1}$ (min) for Tray-2 and between $377 \pm 19 \mu \mathrm{Gy} \mathrm{d}^{-1}$ (max) and $292 \pm 10 \mu \mathrm{Gy} \mathrm{d}^{-1}$ (min) for Tray-3. As stated before these daily absorbed dose values have to be seen as lower limits due to the fact, that the EXPOSE-R facility was only positioned outside the ISS for $82 \%$ of the mission time, and the dose values inside the ISS are lower than for the outside exposure. Nevertheless one can compare the daily dose values measured during the EXPOSE-R mission with a similar experiment performed during the EXPOSE-E mission (Berger et al. 2012; Rabbow et al. 2012). During EXPOSE-E TLDs were also exposed in acrylic glass holders below the dark samples, with an overall mission duration of 583 days and an exposure time outside the Columbus Laboratory of 559 days. The average absorbed dose rates measured with TLDs for EXPOSE-E varied between $207 \pm 12 \mu \mathrm{Gy} \mathrm{d}^{-1}$ (min) and $368 \pm 27 \mu \mathrm{Gy} \mathrm{d}^{-1}$ (max) at similar detector locations as for EXPOSE-R. This dose region agrees quite well with the given measured dose values for the EPXOSE-R mission. Further on one can compare the EXPOSE-R data measured with the TLDs with the data provided by the less shielded R3DR instrument (Dachev et al. 2014). Dachev et al. 2014 gives a total daily dose rate of $675.4 \mu \mathrm{Gy}$. Taking into account, that the R3DR instrument was less shielded than the TLDs and therefore saw a higher contribution from trapped particles almost factor of 2 increase in dose from the bottom to the top of the EXPOSE-R facility is well explained. The dose values measured with the TLDs inside EXPOSE-R are very close to dose values measured inside the ISS (Lishnevskii et al. 2010; Welton et al. 2012; Berger et al. 2013; Pálfalvi et al. 2014) and reflect therefore the high amount of shielding of the biological samples located within the EXPOSE-R facility.

\section{Acknowledgements}

The Austrian participation in this experiment was supported by the Austrian Space Applications Programme (ASAP) of the Federal Ministry for Transport, Innovation and Technology under contract no. 819643. The Polish contribution to this work was supported by the Ministry of Science and Higher Education, grant numbers DWM/N118/ESA/2008 and N N505 261535.

\section{REFERENCES}

Benton, E.R. \& Benton, E.V. (2001). Space radiation dosimetry in low-Earth orbit and beyond. Nucl. Instrum. Methods B. 184, 255-294.

Berger, T. (2008). Radiation dosimetry onboard the International Space Station ISS. Z. Med. Phys. 18, 265-275.

Berger, T. \& Hajek, M. (2008). TL-efficiency - overview and experimental results over the years. Radiat. Meas. 43, 146-156.

Berger, T., Hajek, M., Bilski, P., Koerner, C., Vanhavere, F. \& Reitz, G. (2012). Cosmic radiation exposure of biological test systems during the EXPOSE-E mission. Astrobiology 12, 387-392.

Berger, T., Bilski, P., Hajek, M., Puchalska, M. \& Reitz, G. (2013). The MATROSHKA experiment: results and comparison from EVA (MTR-1) and IVA (MTR-2A/2B) exposure. Radiat. Res. 180(6), 622-637. Bilski, P., Berger, T., Hajek, M. \& Reitz, G. (2011). Comparison of the response of various TLDs to cosmic radiation and ion beams: current results of the HAMLET project. Radiat. Meas. 46, 1680-1685.

Bilski, P., Berger, T., Hajek, M., Twardak, A., Koerner, C. \& Reitz, G. (2013). Thermoluminescence fading studies: implications for long-duration space measurements in Low Earth Orbit. Radiat. Meas. 56, 303-306.

Dachev, T., Horneck, G., Häder, D.-P., Schuster, M. \& Lebert, M. (2014). EXPOSE-R cosmic radiation time profile. Int. J. Astrobiol. (this issue), 
Published online: 12 Mai 2014, http://dx.doi.org/10.1017/ S1473550414000093.

Facius, R. \& Reitz, G. (2006). Space weather impacts on space radiation protection. In Space Weather-Physics and Effects, ed. Bothmer, V. \& Daglis, I.A., pp. 289-353. Springer, Heidelberg.

Goossens, O., Vanhavere, F., Leys, N., De Boever, P., O'Sullivan, D., Zhou, D., Spurný, F., Yukihara, E.G., Gaza, R. \& McKeever, S.W.S. (2006). Radiation dosimetry for microbial experiments in the International Space Station using different etched track and luminescent detectors. Radiat. Prot. Dosim. 120, 433-437.

Hajek, M., Berger, T., Vana, N., Fugger, M., Pálfalvi, J.K., Szabó, J., Eördögh, I., Akatov, Y.A., Arkhangelsky, V.V. \& Shurshakov, V.A. (2008). Convolution of TLD and SSNTD measurements during the BRADOS-1 experiment onboard ISS (2001). Radiat. Meas. 43, 12311236.

Hovestadt, D., Gloeckler, G., Fan, C.Y., Fisk, L.A., Ipavich, F.M., Klecker, B., O'Gallagher, J.J. \& Scholer, M. (1978). Evidence for solar wind origin of energetic heavy ions in the Earth's radiation belt. Geophys. Res. Lett. 5 (12), 1055-1057.

Lishnevskii, A.E., Panasyuk, M.I. \& Benghin, V.V. (2010). Variations of radiation environment onboard the ISS in the Year 2008. Cosmic Research 48, 206-210.

Pálfalvi, J.K. et al. (2014). Passive dosimetry in the Service (Zvezda) module: 2010-2014 In 19th Workshop on Radiation Monitoring for the International Space Station, Krakow, Poland, 9-11 September 2014. http://www.wrmiss.org.
Rabbow, E. et al. (2012). EXPOSE-E: an ESA astrobiology mission 1.5 years in space. Astrobiology 12, 374-386.

Rabbow, E. et al. (2014). The astrobiological mission EXPOSE-R on board of the International Space Station. Int. J. Astrobiol. (this issue), Published online: 28 August 2014 http://dx.doi.org/10.1017/S1473550414000202.

Reitz, G. (2008). Characteristic of the radiation field in low earth orbit and in deep space. Z. Med. Phys. 18, 233-243.

Reitz, G., Beaujean, R., Benton, E., Burmeister, S., Dachev, T., Deme, S., Luszik-Bhadra, M. \& Olko, P. (2005). Space radiation measurements onboard ISS - the DOSMAP experiment. Radiat. Prot. Dosim. 116, 374-379.

Reitz, G., Berger, T., Bilski, P., Facius, R., Hajek, M., Petrov, V., Puchalska, M., Zhou, D., Bossler, J., Akatov, Y. et al. (2009). Astronaut's organ doses inferred from measurements in a human phantom outside the International Space Station. Radiat. Res. 171, 225-235.

Vanhavere, F., Genicot, J.L., O'Sullivan, D., Zhou, D., Spurný, F., Jadrníčková, I., Sawakuchi, G.O. \& Yukihara, E.G. (2008). Dosimetry of biological experiments in space (DOBIES) with luminescence (OSL and TL) and track etch detectors. Radiat. Meas. 43, 694-697.

Welton, A., Gaza, R., Dunegan, A., Barzilla, J., Fry, D., Lee, K. \& Semones, E. (2012). ISS Radiation Measurements 2008-2011 17th Workshop on Radiation Monitoring for the International Space Station, Austin, Texas, USA, September 4-6 2012. http://www.wrmiss.org/ workshops/seventeenth/Welton.pdf.

Yasuda, N., Uchihori, Y., Benton, E.R., Kitamura, H. \& Fujitaka, K. (2006). The intercomparison of cosmic rays with heavy ion beams at NIRS (ICCHIBAN) project. Radiat. Prot. Dosim. 120, $414-420$. 